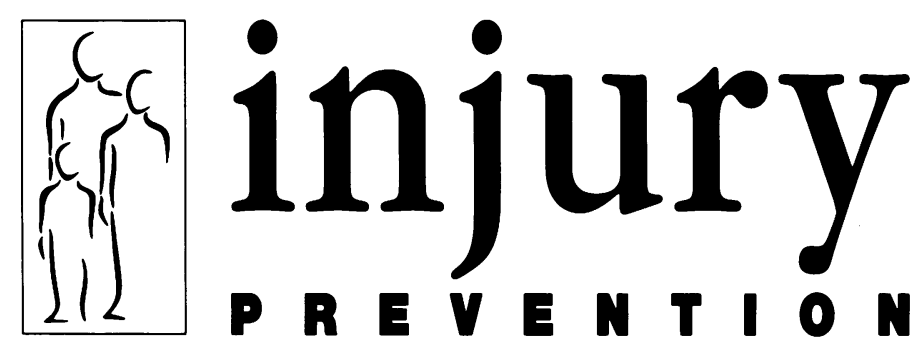

Journal of the International Society for Child and Adolescent Injury Prevention

Editorials

\title{
Descriptive surveys - are they useful?
}

In this issue, as in previous issues, there are a number of papers that present the results of essentially descriptive surveys. Other papers deal with surveillance which is not quite the same as one or more surveys. In both cases we may ask: What do these contribute to injury prevention? A general answer is - far more than most may realize. Readers need to be reminded that few surveys are exclusively descriptive. Most include an analytic component, by which I mean they provide breakdowns or analyses of the injury under scrutiny by other features - age and sex most commonly, but often by many others as well. Occasionally, these analyses are interpreted as identifying 'risk factors', and to some extent this may be correct. It is, however, this 'second level' of analysis - one that goes beyond simply estimating magnitude to identifying groups at greatest risk, for example, that makes many otherwise apparently simple descriptive studies so interesting and potentially valuable.

Depending on what is actually surveyed, or how it is analyzed, most such studies provide both an estimate of the size of a problem and its severity. For example, a survey of playground injuries that includes all medically attended visits as well as hospital admissions, gives a rough approximation of this problem from both perspectives.

Although some surveys focus only on a specific problem, such as head injuries, most are inclusive - that is, they record all injuries regardless of origin, seen in a particular setting over a specified period. If the results are to be used to influence program priorities, it is important to know not only how many of each type of injury are seen, but also the proportions in each category that are serious - whether measured by deaths, disability, hospital or emergency room admissions, or simply as doctor visits. When both numbers and severity are taken into consideration, alongside their preventability, a program priority or target should readily emerge.

For example, the paper by Walsh et al (p 16), pointing so clearly to the dominance of head injuries, cannot but help not only those in the Newcastle community focus still more attention on this problem, but should also reinforce the work of others already concentrating on it. Learning further that injury admissions of all kinds, regardless of severity, are relatively little affected by age, is also a sobering and perhaps unexpected discovery. Equally unexpected is the finding reported by Sacks et al (p 52) that nearly three persons per 1000 are bitten by dogs and that children have three times the rate of medical visits than adults. Is this a problem we have tended to overlook?

In light of these considerations the journal will continue to welcome descriptive reports. They remain the foundation on which much subsequent research and program decisions are built. In stating our willingness to publish yet another survey, however, my personal view is that survey researchers are obliged to go further. First, they must try to do more than simply send reprints to groups they believe can or should use their results. I am convinced the best way to foster implementation is through face-to-face meetings. An added reason for doing so is that such meetings help both parties better understand the perspectives and problems of the other. A second obligation is to move forward towards increasingly more illuminating types of studies. Descriptions should be followed by analyses of the kind referred to above, in a search for possible risk factors. This step, in turn, leads to still more sophisticated designs, such as case-control studies, that shed more light on possible risk factors. Eventually descriptive research should lead to randomised trials or other methods for evaluating existing or planned interventions. Taken together, these steps are certain to increase our rapidly growing knowledge of 'what works'. The only problem that then remains is how to put this into action. That is where advocacy and political action comes in.

\section{Surveillance}

A frequent complaint of many program persons is that they lack the data needed not only to assess the size of a problem in their community but also whether it is increasing or

declining. Repeated surveys are one way of responding to this entirely legitimate concern. Another, more efficient approach, however, is through the establishment of 
systems of surveillance. The distinction between such systems and surveys is not always appreciated.

As a rule, surveillance cannot hope to provide true population based estimates of injury occurrence. This is so because surveillance systems exist to monitor events and thus reveal new trends or unexpected occurrences. Rarely are these systems based on samples of events that are representative of what is happening in the population in which they are situated. For example, the CHIRPP system described by Stone and Doraiswamy in this issue (p 47), both in Glasgow and across Canada, is hospital based and not all injured children are treated in hospital. Even if it is reasoned that it is only injuries of sufficient severity to require hospital attention that are of interest, those treated in hospitals that are not part of the system will be missed. Thus, those 'captured' by the system are likely to reflect a somewhat biased sample even of all hospital treated injuries. Even in cases such as the NEISS system described by Weiss in this issue, or the UK's admirable home accident (HASS) or leisure accident (LASS) systems, where a studious attempt is made to include a representative sample of hospitals, such problems will inevitably remain.

Accordingly, surveillance results are best used to monitor trends or to make comparisons of injuries within the system itself. Under some circumstances the data may, however, also be used to evaluate program initiatives. Any such analyses are potentially of great value and accordingly, attempts to establish even modest, community based surveillance procedures will, almost inevitably, be rewarded. Moreover, they need not be costly. However, as the experience in Glasgow indicates, much groundwork needs to be laid to ensure cooperation and adequate support. Part of the secret is not to attempt to capture more information than is essential, and, where possible, to make the system fit well into the hospital's ordinary operating procedures - for example, by making it a part of administrative or accounting operations.

\section{The Second BC Injury Prevention Conference}

The Second BC Injury Prevention Conference in Vancouver, BC held Nov 23-25 was inspirational. A dispassionate account of it may make it appear little different than most such conferences. But in this case, perhaps because I went expecting 'more of the same', the experience was surprisingly rewarding. I use this opportunity to convey some of the passion I felt at various moments during this unusual event.

First, I was impressed that it was the second such conference. Too often similar gatherings are one-off affairs; the organizers don't see the need to do it again, or don't have the energy to do so. But by bringing much the same group of persons together at regular intervals, not necessarily annually, a sense of corporate spirit is built. In part this is simply a result of establishing personal contacts with other, kindred spirits.

Second, I was not prepared for the exceptionally diverse range of participants and sponsors. This alone speaks volumes about the extent to which the injury prevention message has infiltrated so many levels of society, including the corporate and government sectors. For example, the co-chair was a fire department official who is responsible for an excellent, mobile, nearly full scale home safety display.

Finally, there were a number of highlights. Apart from a typically solid, straightforward, and sensible presentation by Fred Rivara, and a humorous lunch talk by a fire inspector with a serious message, there was a brief presentation by a representative of the Ministry of Transport. In this she announced a number of no-holds-barred tough road safety measures that fully convinced me that this province is genuinely determined to reduce traffic injuries to pedestrian and cyclists. (It is not, therefore, incidental that $\mathrm{BC}$ is the first province to have a mandatory bicycle helmet law with teeth.)

Perhaps this talk should not have been such a surprise because this province has a brief but impressive record of taking injury prevention seriously. For several years BC has had an Office for Injury Prevention (OIP) in the Ministry of Health. This reflects, at least in part, the point I struggled to make in an earlier editorial about injury prevention being a health responsibility. At least this constituency appears to agree.

Continuing on my note of pleasant surprise, the second edition of a directory produced by the OIP lists more than 140 organizations and agencies involved in injury prevention. They cover the alphabet: from the Aboriginal Health Association of BC, BC Automobile Association and BC Children's Hospital's, Safe Start injury prevention program to McDonald's Restaurants, the National Bicycle Safety Foundation, police and fire departments, the Vancouver Health Department and the Whistler Sports Injury Prevention Program. Many of these were represented at the conference itself.

I admit I have had occasion to point to lists such as this and say that with this many players the need for coordination is immense and that someone or some group must take the lead. British Columbia's Minister of Health, the Honourable Paul Ramsey, has done just that, appointing the Minister's Injury Prevention Advisory Committee with the mandate to develop a coordinated comprehensive approach in order to reduce unintentional injuries in British Columbia. The OIP as secretariat plays a supporting role.

All right. It rained for three days and there were some disappointing moments. Far and away the greatest disappointment was my realization that apart from a handful of the speakers there were few, if any, attendees who were health professionals. I will refrain from editorializing further about what I think this means save to say that many health professionals still fail to understand the nature and seriousness of the injury epidemic. 